\title{
Theoretical analysis of sessile evaporating droplet on curved substrate with interfacial cooling effect
}

\author{
Yang Shen ${ }^{1}$, Yongpan Cheng ${ }^{1, *}$, Jinliang $\mathrm{Xu}^{1,{ }^{*}}$, Kai Zhang ${ }^{2}$, Yi Sui ${ }^{3}$.
}

1. Beijing Key Laboratory of Multiphase Flow and Heat Transfer for Low Grade Energy, North China Electric Power University, Beijing 102206, China

2. Beijing Key Laboratory of Emission Surveillance and Control for Thermal Power Generation, North China Electric Power University, Beijing 102206, China

3. School of Engineering and Materials Science, Queen Mary University of London, Mile End Road, London, E1 4NS, United Kingdom.

\section{Accepted by}

\section{Langmuir}

May 2020

\footnotetext{
*Correspondence should be addressed to: Prof. Yongpan Cheng, email: chengyp@ncepu.edu.cn, or Prof. Jinliang Xu, email: xj1@ncepu.edu.cn
} 


\section{Abstract}

Sessile droplet evaporation is widely encountered in nature, and it has numerous applications in industrial and scientific communities, therefore, the accurate prediction of droplet evaporation has great significance in practical applications. In this paper, for the first time, a comprehensive theoretical model is built up for diffusion-controlled heat and mass transfer for sessile droplet evaporation on curved substrate in toroidal coordinate. The evaporative mass transfer is coupled with the heat transfer across the gas-liquid droplet interface, as well as the heat transfer across the solid-liquid interface of the curved substrate. The effects of interfacial cooling, thermal conductivity of droplet and substrate as well as their initial shapes on the droplet evaporation are provided in details. It is found that the evaporative flux usually increases sharply near the droplet edge due to the short distance for heat conduction from the substrate to the droplet, however, it can be reversed from sharp increasing to decreasing at low thermal conductivity ratio $k_{R}<0.3$ of substrate over droplet or large initial droplet contact angle $\theta_{C A}>30^{\circ}$. The interfacial evaporative cooling effect can always suppress the droplet evaporation. The lifetime of evaporative droplet can be prolonged with the decreasing thermal conductivity ratio, increasing evaporative cooling number, increasing initial droplet contact angle or tangential angle of curved substrate. These findings may be of great significance in the applications of droplet evaporation on the curved substrate.

Keywords: Droplet evaporation; Curved substrate; Evaporative cooling; Theoretical analysis; 


\section{Introduction}

Evaporation of liquid droplets on underlying surfaces is omnipresent in our daily life, and it also has numerous industrial and scientific applications [1], such as ink-jet printing [2], DNA chip manufacturing [3], spray cooling [4], virus spreading and test [5]. Thus, a full understanding of the evolution of droplet evaporation is essential for the wide applications. So far extensive experimental and theoretical approaches have been carried out in this area. As the pioneers, Picknet and Bexon [6] identified two extreme modes of evaporation of a droplet on the substrate in still air, namely the Constant Contact Radius (CCR) mode and Constant Contact Angle (CCA) mode. In CCR mode the contact radius remains constant with decreasing contact angle, and in CCA mode the contact angle remains constant with decreasing contact radius.

In order to carry out the theoretical studies on drop evaporation phenomenon, the simplifications are needed to get the idealized model [7]. The isothermal quasi-steady assumptions are usually made [8-10], in which the temperature along the gas-liquid interface is uniform, and vapor concentration at the interface is at the saturation value at the uniform temperature, the evaporation is driven by the vapor diffusion around the droplet according to the Fick's law. Based on the lubrication theory, Deegan et al [11] investigated the evaporation of pinned sessile drops and revealed the mechanism of "coffee-ring" formation on hydrophilic substrate. With the help of Finite Element Analysis, $\mathrm{Hu}$ and Larson [12] derived the expression for overall evaporation rate for contact angle $\theta \leq 90^{\circ}$, which agrees quite well with the experimental data. Bhardwaj [13] extended the study to droplet evaporation on hydrophobic substrate for contact angle $90^{\circ}<\theta \leq 180^{\circ}$. Due to the similarity between the vapor concentration field around the droplet and electrostatic potential field of conductor, according to the 
available electrostatic potential field by Lebedev [14], Popov [15] obtained the vapor concentration field around the droplet, as well as the evaporation rate and evaporation mass flux at arbitrary droplet contact angles. Based on the expressions, Nguyen and Nguyen derived the lifetime of sessile evaporating droplet in CCA and CCR modes with the effect of contact angle, contact radius and droplet height [16], then the power law was provided for the transient variation of droplet volume over time [17]. The theoretical results were further validated through their experiments [18]. Nguyen and Nguyen [19] found that the nanoparticles can increase the overall rate of droplet diffusive evaporation, leading to decrease of the droplet lifetime. Additionally, they also found that surfactants can affect the liquid cohesive energy density, as well as the droplet evaporation [20].

Due to the uneven evaporation flux around the droplet, the temperature along the gas-liquid interface is not uniform as well as the vapor concentration. Chandramohan et al. [21] adopted the spatiotemporal infrared measurement to obtain the interfacial temperature distribution, as well as the transient variation of droplet volume and contact angle during the droplet evaporation. Dash and Garimella [22] found that due to the evaporative cooling, the actual lifetime of droplet evaporation is longer than that predicted by the isothermal model. $\mathrm{Xu}$ and $\mathrm{Ma}$ [23] simulated the droplet evaporation by coupling the temperature field in the droplet and the vapor concentration field in the atmosphere together, and found that both the evaporation flux along the droplet surface and the total evaporation rate will decrease with increase evaporative cooling effect. By coupling the vapor concentration and temperature field Nguyen et al. [24] derived the expression for evaporative flux around the droplet with the interfacial cooling effect for the first time, which is applicable for a full range of spherical-cap shape droplets of different contact angles 
and types of fluids.

Besides the evaporative cooling effect, the underlying substrate also has great effect on the droplet evaporation. Gleason et al. [25] experimentally studied the water droplet evaporation on a heated substrate, and found that the substrate cooling cannot be neglected for evaporation on polymer substrate with low thermal conductivity. Dunn et al.[26] revealed the strong influence of substrate conductivity on wetting droplet evaporation, and they proposed a mathematical model coupling the vapor concentration in the atmosphere and temperature in the liquid and substrate. Wang et al. [27] simulated the droplet evaporation with combined effects of evaporative cooling and the underlying substrate, and found that the influence of substrate on the droplet evaporation depends greatly on the strength of evaporative cooling. Saenz et al. [28] carried out the direct numerical simulation (DNS) on the non-axisymmetric sessile droplet evaporation, as well as the experimental studies. They found that the interfacial temperature keeps almost constant in the CCA mode, while it increases in the CCR mode as the droplet becomes thinner, therefore, with the increasing substrate heating, the evaporation rate in CCR mode will increase more rapidly than that in CCA mode. Besides the flat substrate, Petsi and Burganos [29] analytically studied the simplified two-dimensional isothermal droplet on curved substrate under various evaporation conditions, and it is found that the temperature drop at the free surface of a droplet on a convex hydrophobic substrate is far greater than that for flat or concave substrates of the same hydrophobicity.

From the literature review above, it can be seen that most of the studies are focusing on the droplet evaporation on the flat substrate, and few studies are on droplet evaporation on the curved substrate. In reality, the curved substrate is more widely encountered than the flat substrate. For curved substrate the heat for interfacial 
evaporation is transported through both substrate and droplet, the heat conduction through the curved substrate will have great effect on droplet evaporation. Furthermore, the interfacial cooling, vapor diffusion around the droplet will also affect the droplet evaporation, and these processes are strongly coupled together. So far the studies in this area are still scarce. Therefore, in this paper the theoretical analysis will be carried out for droplet evaporation on three-dimensional curved substrate, the evaporative mass transfer at the droplet interface is coupled with the heat transfer inside the substrate and droplet. The results can provide the theoretical guidance for numerical and experimental studies, and they are of great significance for the wider applications of sessile droplet evaporation.

\section{Theoretical Model}

For simplification the curved substrate is assumed as the shape of spherical cap, with the tangential angle $\theta_{\mathrm{Sub}}$ at the edge. When the gravitational effect is ignored, the droplet surface is also of the shape of spherical cap, with the contact angle $\theta_{\mathrm{CA}}$ on the substrate, $\theta=\theta_{\mathrm{CA}}+\theta_{\text {Sub }}$ is the cutting angle of droplet edge over the horizontal substrate bottom. Therefore, the droplet on the curved substrate can be exactly mapped in toroidal coordinate $(\alpha, \beta)$, which is a three-dimensional orthogonal coordinate that results from rotating the two-dimensional bipolar coordinate system about the axis that separates its two foci, as shown in Figure 1. The relationship between the toroidal coordinate $(\alpha, \beta)$ and the cylindrical coordinate $(r, z)$ is shown below.

$$
r / \sinh \alpha=z / \sin \beta=R(\cosh \alpha-\cos \beta)^{-1}
$$

where $R$ is base radius of droplet. 
The bottom of the substrate is assumed as constant temperature, the heat will be transferred to the gas-liquid interface for evaporation through substrate and droplet sequentially. The convective heat transfer inside the droplet is ignored, hence only heat conduction occurs inside the droplet and substrate, the temperature field is controlled by Laplace's equation $\nabla^{2} T=0$. Outside the droplet the vapor concentration field is diffusion-controlled without convective effect, hence it is controlled by Laplace equation $\nabla^{2} C=0$. The detailed derivations can be referred to the supplementary materials, here the brief introduction is shown below.

The boundary conditions of vapor concentration and temperature are as follows:

For vapor region around the droplet $(0 \leq \alpha<\infty, 2 \pi \leq \beta \leq 3 \pi-\theta)$

(1) In the region far from the droplet, temperature and vapor concentration are $T_{\infty}, C_{\infty}$ respectively

(2) At the axis of symmetry:

$$
(\partial C(\alpha, \beta) / \partial \alpha)_{\alpha=0}=0
$$

(3) At the gas-solid interface, no penetration for vapor into the solid substrate:

$$
(\partial C(\alpha, \beta) / \partial \beta)_{\beta=2 \pi}=0
$$

(4) At the gas-liquid interface, the heat transfer is coupled with the evaporative mass transfer, i.e. evaporative cooling effect is considered.

$$
(q(\alpha))_{\beta=\pi-\theta}=L(J(\alpha))_{\beta=3 \pi-\theta}
$$

where $q(\alpha)$ is heat flux, $L$ is liquid latent heat of vaporization, $J(\alpha)$ is evaporative flux.

For temperature region within the $\operatorname{droplet}\left(0 \leq \alpha<\infty, \pi-\theta \leq \beta \leq \pi-\theta_{\text {Sub }}\right)$

(1) At the axis of symmetry:

$$
\left(\partial T_{\mathrm{L}}(\alpha, \beta) / \partial \alpha\right)_{\alpha=0}=0
$$


(2) At the solid-liquid interface, the heat transfer inside the substrate and droplet is coupled together, hence there is no temperature jump across the interface.

$$
T_{\mathrm{L}}\left(\alpha, \pi-\theta_{\mathrm{Sub}}\right)=T_{\mathrm{S}}\left(\alpha, \pi-\theta_{\mathrm{Sub}}\right)
$$

The heat flux is identical from both sides

$$
\left.\frac{(\cosh \alpha-\cos \beta)}{R} \frac{\partial T_{\mathrm{L}}(\alpha, \beta)}{\partial \beta}\right|_{\beta=\pi-\theta_{\text {sub }}}=\left.k_{R} \frac{(\cosh \alpha-\cos \beta)}{R} \frac{\partial T_{\mathrm{S}}(\alpha, \beta)}{\partial \beta}\right|_{\beta=\pi-\theta_{\text {Sub }}}
$$

where $k_{\mathrm{R}}=k_{\mathrm{S}} / k_{\mathrm{L}}$ is relative thermal conductivity of substrate and droplet.

For temperature region within the substrate $\left(0 \leq \alpha<\infty, \pi-\theta_{\text {Sub }} \leq \beta \leq \pi\right)$

(1) At the axis of symmetry:

$$
\left(\partial T_{\mathrm{S}}(\alpha, \beta) / \partial \alpha\right)_{\alpha=0}=0
$$

(2) On the bottom, the temperature is constant

$$
T_{\mathrm{S}}(\alpha, \pi)=T_{\mathrm{W}}
$$

According to Nguyen et al.[24], the normalized vapor concentration field around the droplet can be written:

$$
\widetilde{C}(\alpha, \beta)=\frac{C(\alpha, \beta)-C_{\propto}}{C_{\mathrm{e}}-C_{\propto}}=\sqrt{2 \cosh \alpha-2 \cos \beta} \int_{0}^{\infty} E_{\mathrm{C}}(\tau) P_{i \tau-0.5}(\cosh \alpha) \cosh [(2 \pi-\beta) \tau] d \tau
$$

Similarly it can be derived that the normalized temperature field within the droplet is

$$
\begin{aligned}
& \widetilde{T}_{L}(\alpha, \beta)=\frac{T_{\mathrm{L}}(\alpha, \beta)-T_{\mathrm{W}}}{T_{\mathrm{W}}-T_{\infty}}=\sqrt{2 \cosh \alpha-2 \cos \beta} \\
& \int_{0}^{\infty} P_{i \tau-0.5}(\cosh \alpha) E_{\mathrm{S}}(\tau)\left\{-H\left(\theta_{\mathrm{Sub}}, \tau\right) \sinh \left[\left(\pi-\theta_{\mathrm{Sub}}-\beta\right) \tau\right]+\sinh \left(\theta_{\mathrm{Sub}} \tau\right) \cosh \left[\left(\pi-\theta_{\mathrm{Sub}}-\beta\right) \tau\right]\right\} d \tau
\end{aligned}
$$

The temperature field within substrate is

$$
\widetilde{T_{\mathrm{S}}}(\alpha, \beta)=\frac{T_{\mathrm{S}}(\alpha, \beta)-T_{\mathrm{W}}}{T_{\mathrm{W}}-T_{\infty}}=\sqrt{2 \cosh \alpha-2 \cos \beta} \int_{0}^{\infty} E_{\mathrm{S}}(\tau) P_{i \tau-0.5}(\cosh \alpha) \sinh [(\pi-\beta) \tau] d \tau
$$

where $\tau, P_{i \tau-0.5}(\cosh \alpha)$ and $C_{\mathrm{e}}$ are the integration dummy, Legendre functions of the first kind and the saturated vapor concentration of the liquid at temperature $T_{\mathrm{W}}$ of the 
substrate bottom respectively.

$E_{\mathrm{C}}(\tau)$ and $E_{\mathrm{S}}(\tau)$ are functions of the integration dummy, and are independent of the toroidal coordinates $\alpha$ and $\beta$

$$
\begin{aligned}
& E_{\mathrm{C}}(\tau)=\frac{\cosh (\theta \tau)}{\cosh (\pi \tau)} \times \\
& \frac{\operatorname{sech}[(\theta-\pi) \tau]\left(\frac{f\left(k_{\mathrm{R}}, \tau\right)}{3} \frac{\partial F(\theta, \tau)}{\partial \theta}-\tau F(\theta, \tau) g\left(k_{\mathrm{R}}, \tau\right)\right)}{\left\{\frac{f\left(k_{\mathrm{R}}, \tau\right)}{3} \frac{\partial F(\theta, \tau)}{\partial \theta}-\tau F(\theta, \tau) g\left(k_{\mathrm{R}}, \tau\right)\right\}-E_{0}\left\{\frac{\partial F(\theta, \tau)}{3 \partial \theta}-\tau F(\theta, \tau) \tanh [(\theta-\pi) \tau]\right\} f\left(k_{\mathrm{R}}, \tau\right)} \\
& E_{\mathrm{S}}(\tau)=\frac{E_{0} \cosh (\theta \tau)}{\cosh (\pi \tau)} \times \\
& \frac{\frac{\partial F(\theta, \tau)}{3 \partial \theta}-\tau F(\theta, \tau) \tanh [(\theta-\pi) \tau]}{\left\{\frac{f\left(k_{\mathrm{R}}, \tau\right)}{3} \frac{\partial F(\theta, \tau)}{\partial \theta}-\tau F(\theta, \tau) g\left(k_{\mathrm{R}}, \tau\right)\right\}-E_{0}\left\{\frac{\partial F(\theta, \tau)}{3 \partial \theta}-\tau F(\theta, \tau) \tanh [(\theta-\pi) \tau]\right\} f\left(k_{\mathrm{R}}, \tau\right)}
\end{aligned}
$$

where

$$
\begin{aligned}
& H\left(\theta_{\mathrm{Sub}}, \tau\right)=\frac{\frac{d F\left(\theta_{\mathrm{Sub}}, \tau\right)}{d \theta_{\mathrm{Sub}}}\left(k_{\mathrm{R}}-1\right) \sinh \left(\theta_{\mathrm{Sub}} \tau\right)}{3 \tau F\left(\theta_{\mathrm{Sub}}, \tau\right)}-k_{\mathrm{R}} \cosh \left(\theta_{\mathrm{Sub}} \tau\right) \\
& F(\theta, \tau)=\sin (\theta \tau) /[\sinh (\pi \tau) \sin \theta]
\end{aligned}
$$

According $\mathrm{Xu}$ and $\mathrm{Ma}[23]$, the non-dimensional evaporative cooling number is defined as

$$
E_{0}=b L D / k_{L}
$$

where $b$ is the thermal gradient of vapor saturation concentration over temperature $\mathrm{d} C_{\text {sat }} / \mathrm{d} T, L$ is liquid latent heat of vaporization, $D$ is coefficient of vapor diffusion in air, $k_{\mathrm{L}}$ is liquid thermal conductivity. $E_{0}$ reflects the intensity of the effect of the evaporative cooling on the droplet evaporation, and is the ratio of the reduction in the evaporation flux to its initial value. It is noted that larger $E_{0}$ means more significant negative feedback effect of evaporative cooling to reduce evaporation rate. At $E_{0}=0$ 
it is the isothermal model with the uniform temperature around the droplet without evaporative cooling effect.

The evaporation flux at the droplet surface is

$$
J(\alpha, \theta)=\frac{\mathrm{D}\left(C_{\mathrm{e}}-C_{\propto}\right) \sqrt{2}(\cosh \alpha+\cos \theta)^{3 / 2}}{R} \int_{0}^{\infty} E_{\mathrm{C}}(\tau) P_{i \tau-0.5}\left\{\frac{\cosh [(\theta-\pi) \tau] \sin \theta}{2(\cosh \alpha+\cos \theta)}-\tau \sinh [(\theta-\pi) \tau]\right\} d \tau(10)
$$

Integrating it over the droplet surface in toroidal coordinates, the evaporation rate is.

$$
\frac{d m(\theta)}{d t}=-D\left(C_{\mathrm{e}}-C_{\propto}\right) 2 \sqrt{2} \pi R \varphi\left(\theta, k_{\mathrm{R}}, E_{0}\right)
$$

where

$$
\begin{aligned}
& \varphi\left(\theta, k_{\mathrm{R}}, E_{0}\right)= \\
& \int_{0}^{\infty} d \tau \int_{0}^{\infty}(\cosh \alpha+\cos \theta)^{-0.5} \sinh \alpha\left[E_{\mathrm{C}}(\tau) P_{i \tau-0.5}(\cosh \alpha)\left[\frac{\cosh [(\theta-\pi) \tau] \sin \theta}{2(\cosh \alpha+\cos \theta)}-\tau \sinh [(\theta-\pi) \tau]\right]\right] d \alpha
\end{aligned}
$$

The volume of the droplet is

$$
V=\frac{\pi R^{3}}{3 g(\theta)}-\frac{\pi R^{3}}{3 g\left(\theta_{\mathrm{Sub}}\right)}
$$

where

$$
g(\theta)=\frac{\sin ^{3} \theta}{(1-\cos \theta)^{2}(2+\cos \theta)}
$$

When the droplet evaporates in CCR mode, the base radius is kept constant while the contact angle is gradually decreases. Combining the Eq.(11) and Eq.(13), the variation of contact angle over time can be obtained.

$$
\frac{d \theta}{d t}=-\frac{D\left(C_{\mathrm{e}}-C_{\propto}\right)}{\rho_{\mathrm{L}} R^{2}} 2 \sqrt{2} \varphi\left(\theta, k_{\mathrm{R}}, E_{0}\right)(1+\cos \theta)^{2}
$$

The lifetime of droplet in CCR mode can be integrated to give.

$$
t_{\mathrm{CCR}}=\frac{\rho_{\mathrm{L}} R_{0}{ }^{2}}{2 \sqrt{2} D\left(C_{\mathrm{e}}-C_{\propto}\right)} \int_{\theta_{\text {sub }}}^{\theta_{0}} \frac{1}{\varphi\left(\theta, k_{\mathrm{R}}, E_{0}\right)(1+\cos \theta)^{2}} d \theta
$$

where the $\theta_{0}$ and $R_{0}$ are the initial tangential angle of the droplet surface with the plane at the edge and initial base radius respectively. 


\section{Results and Discussion}

\subsection{Validation of current analytical model.}

In isothermal model of droplet evaporating without evaporative cooling effect, the temperature around the droplet surface is kept constant. However, for real droplet evaporation, due to the interfacial evaporative cooling effect, the temperature at the droplet surface will be lowered, furthermore, the temperature will not be constant due to the variable evaporation flux around the droplet. In the meantime, the lower temperature will lead to the lower evaporation flux at the surface, leading to longer lifetime of evaporating droplet, as proved by Dash and Garimella [22] that the isothermal model can over-predict the evaporation rate by $20 \%$. In order to validate our analytical model with evaporative cooling effect, the evaporation rates from our model and experiments by Dunn et al.[26,30] are compared for three types of liquid droplets (water, methanol and acetone) at the flat substrate. In their experiments the droplets are generated through the syringe pump and deposited gently on the substrate. The droplet shape is captured with the charge-coupled device (CCD) camera, and analyzed with droplet shape analysis (DSA) software. In their experiments, the evaporating droplets have a volume ranging from 0.5 to $8 \mu \mathrm{m}$, and the base radius ranging from 0.7 to $1.8 \mathrm{~mm}$, the initial contact angle is $40^{\circ}$ for acetone, $43^{\circ}$ for methanol and $60^{\circ}$ for water. The relative humidity is $40 \%$ for water and zero for acetone and methanol with the ambient temperature $T_{0}=295 \mathrm{~K}$ and ambient pressure $P_{0}=99.8 \mathrm{kPa}$. The resultant evaporative cooling numbers $E_{0}$ for water, methanol and acetone are $0.11,0.84$ and 1.03 respectively. During the droplet evaporation the contact angle will monotonically decrease with time, the evaporation rate is calculated 
based on the average values over the variation range of contact angles. In Fig.2 the predicted overall evaporative rates in our non-isothermal model are compared with the experimental measurement by Dunn et al[26, 30]. It is found that the predicted results agree quite well with the experimental results for three types of liquid droplets with different evaporative cooling effects.

\subsection{Temperature field inside the evaporating droplet.}

According to Eq.(3) and Eq.(4) the normalized temperatures inside the droplet and substrate can be calculated respectively. In Fig. 3 the temperature fields are shown under different ratios $k_{R}$ of thermal conductivities of substrate over droplet at evaporative cooling number $E_{0}=1$, tangential angle of curved substrate $\theta_{\text {Sub }}=40^{\circ}$ and droplet contact angle $\theta_{\mathrm{CA}}=50^{\circ}$. According to the definition of normalized temperature in Eq.(3) and Eq.(4), when the temperature is close to the wall temperature $T_{\mathrm{w}}$, the normalized temperature is close to 0 ; when the temperature is close to the ambient temperature $T_{\infty}$, the normalized temperature is close to -1 . It can be clearly seen that the relative thermal conductivity has an important effect on the temperature field. When the thermal conductivity ratio is large at $k_{R}=5$, the thermal resistance in the substrate is lower than that in the droplet, hence the temperature inside the substrate is more uniform, the temperature drop is mainly inside the droplet. When the ratio becomes infinity, the substrate will be at the same temperature $T_{\mathrm{w}}$ as the bottom. However, if the thermal conductivity is low at $k_{R}=0.5$, the temperature drop is mainly inside the substrate, and the temperature inside the droplet become more uniform than that inside the substrate, the droplet surface temperature is around -0.55 , lower than -0.40 at $k_{R}=5$. 


\subsection{Interfacial distribution of evaporative fluxes and temperatures}

Fig.4 shows the distribution of evaporative fluxes and temperatures along the gas-liquid interface under different ratios $k_{R}$ of thermal conductivities of substrate over droplet at evaporative cooling number $E_{0}=1$, tangential angle of curved substrate $\theta_{\mathrm{Sub}}=40^{\circ}$ and droplet contact angle $\theta_{\mathrm{CA}}=50^{\circ}$. It is found that in most of the region near the droplet center, the evaporative flux is quite uniform. Near the edge of the droplet, at the thermal conductivity ratio $k_{R} \geq 0.3$, the evaporative flux will increase sharply. Because at the edge the droplet surface is quite close to the substrate bottom, the heat can be easily transferred to the surface for evaporation, the evaporative cooling effect is not so significant, thus the temperature near the edge is increasing dramatically, close to the ambient temperature. The high temperature can result in the high evaporative flux near the edge, as for the droplet evaporation under isothermal condition when the contact angle is $\theta_{0}<90^{\circ}$. However, when the thermal conductivity ratio $k_{R}<0.3$, the evaporation flux gradually decreases to zero near edge, because at low thermal conductivity of substrate, the heat cannot be transferred to the droplet surface near the edge to compensate for the heat loss due to evaporation, thus the temperature near the edge will be reduced greatly, as seen in Fig. 4(b), leading to low evaporation flux. It is also found that with the increasing thermal conductivity ratio $k_{R}$, the evaporative flux near the center of droplet $r=0$ will increase. For example, when $k_{R}$ increases ten times from 1 to 10 , the evaporative fluxes increase from 0.44 to 0.54 . At low value of $k_{R}$, the effect is more significant, when $k_{R}$ decreases from 1 to 0.1 , the evaporative flux decrease from 0.44 to 0.18 . 
Fig. 5 shows the radial variation of normalized heat fluxes, interfacial temperatures along the substrate surface under different ratios $k_{R}$ of thermal conductivities of substrate over droplet at evaporative cooling number $E_{0}=1$, tangential angle of curved substrate $\theta_{\mathrm{Sub}}=40^{\circ}$ and droplet contact angle $\theta_{\mathrm{CA}}=50^{\circ}$. It can be seen obviously from Fig.5(a) that regardless of the value of thermal conductivity ratio, along the radial direction the heat flux will increase mildly first, then increase rapidly near the edge, because near the edge the heat conduction distance becomes short from the substrate bottom, the heat can be easily transferred to the solid-liquid interface. Furthermore, with the increasing thermal conductivity ratio, the heat flux from the substrate to the droplet will be increased.

From Fig.5(b), it can be found that the interfacial temperature along the substrate surface is quite uniform in most of the region near the center. When $k_{R} \geq 0.3$ the temperature near the edge increases sharply, while at $k_{R}=0.1$ the temperature near the edge is reduced due to the decreased temperature in the droplet edge. When the thermal conductivity ratio is low at $k_{R}=0.1$, the normalized interfacial temperature is quite low, i.e. the substrate temperature is near the ambient temperature, because the larger thermal conductivity in the droplet leads to its uniform temperature distribution in the droplet. When the thermal conductivity ratio is high at $k_{R}=10$, the normalized temperature is near 0 , i.e. the substrate temperature is near the bottom temperature.

Fig. 6 shows the radial distribution of normalized evaporative fluxes, interfacial temperatures along the droplet surface under different droplet contact angles $\theta_{\mathrm{CA}}$ of droplet at evaporation cooling number $E_{0}=1$, thermal conductivity ratio $k_{R}=0.1$ 
and tangential angle of curved substrate $\theta_{\text {Sub }}=40^{\circ}$. It can be seen that the droplet contact angle has significant effect on the evaporative flux and interfacial temperature around the droplet surface. In most of the regions near the droplet center, the evaporative flux is quite uniform, as well as the interfacial temperature. However, near the droplet edge both the evaporative flux and interfacial temperature show quite different characteristics, depending on the droplet contact angle. When the droplet contact angle is low, the evaporative flux is increased sharply near the edge, as well as the interfacial temperature, while when the droplet contact angle is large, both the evaporative flux and interfacial temperature decrease dramatically. The contact angle $\theta_{C A}=30^{\circ}$ is the dividend line for the two different phenomena. Because at the low droplet contact angle, the heat transfer distance from the substrate bottom to the droplet surface is short, heat can be easily supplied to the droplet surface for evaporation, leading to the large evaporative flux and high interfacial temperature near the droplet edge, while at large droplet contact angle, heat is not easy to be supplied to the droplet surface due to long distance.

\subsection{The lifetime of droplet}

For the accuracy of comparison of droplet lifetime, the droplet volumes under different initial contact angles are set identical as those with initial droplet contact angle $\theta_{\text {In }}=90^{\circ}$ on the flat substrate $\theta_{\text {Sub }}=0$, and all the dimensions are normalized with the base radius for the baseline case.

\subsubsection{Effect of thermal conductivity ratio}

Fig.7 shows the transient variation of contact angle $\theta_{\mathrm{CA}}$ of droplet under different ratios $k_{R}$ of thermal conductivities of substrate over droplet at evaporative cooling number $E_{0}=1$ and tangential angle of curved substrate $\theta_{\text {Sub }}=40^{\circ}$. The initial 
contact angle of droplet with the substrate is $\theta_{\mathrm{In}}=50^{\circ}$, during the droplet evaporation the contact angle will decrease until zero when the droplet is dried out. It is clearly seen that as the relative thermal conductivity $k_{R}$ increases, the droplet lifetime will decrease, it is because that the lower thermal conductivity of substrate can transfer less heat to droplet for evaporation, leading to longer lifetime. It is interesting to find that when the thermal conductivity ratio $k_{R}$ is low, it has more profound effect on the droplet lifetime. For example, when $k_{R}$ is increased from 1 to 10 , the lifetime of droplet decreases only a little from 0.56 to 0.46 , however, when $k_{R}$ is increased from 0.1 to 1 , the lifetime decreases dramatically from 2.0 to 0.56 .

\subsubsection{Effect of evaporative cooling}

The evaporative cooling effect can affect the droplet lifetime on the curved substrate, as shown in Fig.8. Transient variations of contact angle of droplet $\theta_{\mathrm{CA}}$ are provided under different evaporative cooing number $E_{0}$ for different thermal conductivity ratio $k_{R}$ at tangential angle of curved substrate $\theta_{\text {Sub }}=40^{\circ}$. Three typical evaporative cooling numbers are selected as $0.11,0.84,1.03$, corresponding to the evaporation of water, methanol and acetone respectively at ambient temperature $T_{0}=295 \mathrm{~K}$ and ambient pressure $P_{0}=99.8 \mathrm{kPa}$. It can be found that the evaporative cooling number has great effect on the droplet lifetime, when the evaporative cooling number is increased, the droplet lifetime will be prolonged, the effect becomes more significant at low thermal conductivity ratio. For example, at the thermal conductivity ratio $k_{R}=10$, when the evaporative cooling number is increased from 0.11 to 1.03 , the lifetime of droplet increases mildly from 0.38 to 0.46 , while at $k_{R}=0.1$, the lifetime of droplet increases dramatically from 1.05 to 2.02 . 


\subsubsection{Effect of initial shape of droplet}

The initial contact angle of droplet can affect the lifetime of the evaporating droplet, as shown in Fig.9, the transient variations of contact angle of droplet $\theta_{\mathrm{CA}}$ are provided under different initial droplet contact angles $\theta_{\mathrm{In}}$ at evaporative cooling number $E_{0}=1$ and tangential angle of curved substrate $\theta_{\text {Sub }}=40^{\circ}$. The initial droplet volume is kept constant under different initial contact angles $\theta_{\mathrm{In}}=10^{\circ}, 30^{\circ}, 50^{\circ}$. It can be found that when the initial contact angle $\theta_{\mathrm{In}}$ is increased, the lifetime is prolonged, because the large initial contact angle can result in long heat transfer distance from the substrate bottom to the droplet surface, the interfacial droplet evaporation is weakened. The effect of the initial contact angle is more significant at low thermal conductivity ratio. For example, at the thermal conductivity ratio $k_{R}=10$, when the initial contact angle increases from $10^{\circ}$ to $50^{\circ}$, the lifetime of droplet increases twice from 0.23 to 0.46 , while at the thermal conductivity ratio $k_{R}=0.1$, the lifetime of droplet increases almost three times from 0.72 to 2.0 .

\subsubsection{Effect of curved substrate shape}

The shape of the curved substrate has great effect on the droplet lifetime, as shown in Fig.10, the transient variations of contact angle of droplet $\theta_{\mathrm{CA}}$ under different tangential angles $\theta_{\text {Sub }}$ of curved substrate are provided at evaporative cooling number $E_{0}=1$. It is noted that the initial tangential angle of droplet over the horizontal plane at the edge is kept at $\theta_{0}=90^{\circ}$, the initial droplet volume is also kept identical. It can be seen that the large tangential angle of curved substrate can lead to long droplet lifetime, because at large tangential angle, the heat will take long 
distance to reach the droplet surface from the substrate bottom, thus the droplet evaporation is weakened. The effect of the tangential contact is more significant at low thermal conductivity ratio. For example, at $k_{R}=10$ droplet lifetime increases from 0.37 to 0.55 when the tangential angle is increased from $20^{\circ}$ to $60^{\circ}$, while at $k_{R}=0.1$, the lifetime is increased from 1.23 to 2.60 .

\section{Conclusion}

In this study the theoretical model for the sessile droplet evaporation on curved substrate is built up in toroidal coordinate, the evaporative mass transfer and heat transfer are coupled together at the gas-liquid interface, as well as the heat transfer at the solid-liquid interface. The effect of key parameters on the droplet evaporation is studied, such as the interfacial evaporative cooling, thermal conductivity ratio of substrate over droplet, the initial droplet contact angle and tangential angle of curved substrate. The main findings are summarized as follows:

1. The evaporative flux and interfacial temperature around the droplet surface are quite uniform in most regions of droplet near the center, while near the droplet edge, they will increase or decrease dramatically due to different local heat transfer performance.

2. The low thermal conductivity ratio $k_{R}<0.3$ or the high initial droplet contact angle $\theta_{C A}>30^{\circ}$ can lead to the sharp decrease of evaporative flux and interfacial temperature near the droplet edge.

3. The lifetime of evaporative droplet can be prolonged with the decreasing thermal conductivity ratio, increasing evaporative cooling number, increasing initial droplet contact angle and tangential angle of curved substrate.

\section{Acknowledgement}


This work was financially supported by the Natural Science Foundation of Beijing

(Grant No: 3162029), Natural Science Foundation of China (Grant No: 51436004),

Marie Curie European Fellowship (Grant No. 658437).

\section{References}

[1] Mampallil, D.; Eral, H. B. A review on suppression and utilization of the coffee-ring effect. Adv. Colloid Interface Sci. 2018, 252, 38-54.

[2] Yoo, H.; Kim, C. Experimental studies on formation, spreading and drying of inkjet drop of colloidal suspensions. Colloid Surf. 2015, 468, 234-245.

[3] Dugas, V.; Broutin, J.; Souteyrand, E. Droplet evaporation study applied to DNA chip manufacturing. Langmuir 2005, 21, 9130-9136.

[4] Kumari, N.; Garimella, S.V. Characterization of the heat transfer accompanying electrowetting or gravity-induced droplet motion. Int. J. Heat Mass Transfer. 2011, $54,4037-4050$.

[5] Zhang, J.; Mahalanabis, M.; Liu, L.; Chang, J.; Pollock, N.; Klapperich, C. A disposable microfluidic virus concentration device based on evaporation and interfacial tension. Diagnostics. 2013, 3, 155-169.

[6] Picknett, R.G.; Bexon, R. The evaporation of sessile or pendant drops in still air. J. Colloid Interface Sci. 1977, 61, 336-350.

[7] Erbil, H. Y. Evaporation of pure liquid sessile and spherical suspended drops: A review. Adv. Colloid Interface Sci. 2012, 170, 67-86.

[8] Deegan, R. D.; Bakajin, O.; Dupont, T. F.; Huber, G.; Nagel, S. R.; Witten, T.A. Capillary flow as the cause of ring stains from dried liquid drops. Nature 1997, $389,827-829$.

[9] Hu, H.; Larson, R. G. Analysis of the microfluid flow in an evaporating sessile 
droplet. Langmuir 2005, 21, 3963-3971.

[10] Stauber, J. M.; Wilson, S. K.; Duffy, B. R. Evaporation of droplets on strongly hydrophobic substrates. Langmuir 2015, 31, 3653-3660.

[11] Deegan, R. D.; Bakajin, O.; Dupont, T. F.; Huber, G.; Nagel, S. R.; Witten, T. A. Contact line deposits in an evaporating drop. Phys. Rev. E. 2000, 62, 756-765.

[12] Hu, H.; Larson, R. G. Evaporation of a sessile droplet on a substrate. J. Phys. Chem. B. 2002, 106, 1334-1344.

[13] Bhardwaj, R. Analysis of an evaporating sessile droplet on a non-wetted surface. Colloid Interface Sci. Commun. 2018, 24, 49-53.

[14] Lebedev, N. N. Special functions and their applications, Revised English ed; Prentice-Hall: Englewood Cliffs, 1965.

[15] Popov, Y.O. Evaporative deposition patterns: spatial dimensions of the deposit. Phys. Rev. E. 2005, 71, 036313.

[16] Nguyen, T. A. H.; Nguyen, A. V. On the lifetime of evaporating sessile droplets. Langmuir 2012, 28, 1924-1930.

[17] Nguyen, T. A. H.; Nguyen, A. V. Transient volume of evaporating sessile droplets: 2/3, 1/1, or another power law? Langmuir 2014, 30, 6544-6547.

[18] Nguyen, T. A. H.; Nguyen, A. V.; Hampton, M. A.; Xu, Z. P.; Huang, L. B.; Rudolph, V. Theoretical and experimental analysis of droplet evaporation on solid surfaces. Chem. Eng. Sci 2012, 69, 522-529.

[19] Nguyen, T. A. H.; Nguyen, A. V. Increased evaporation kinetics of sessile droplets by using nanoparticles. Langmuir 2012, 28, 16725-16728.

[20] Nguyen, T. A. H.; Biggs, S.; Doi, A.; Nguyen, A. V. A new way of assessing droplet evaporation independently of the substrate hydrophobicity and contact line mode: A case study of sessile droplets with surfactants. Colloids Surf. A. 
2019, 577, 396-404.

[21] Chandramohan, A.; Weibel, J. A.; Garimella, S. V. Spatiotemporal infrared measurement of interface temperatures during water droplet evaporation on a nonwetting substrate. Appl. Phys. Lett. 2017, 110, 041605.

[22] Dash, S.; Garimella, S. V. Droplet evaporation dynamics on a superhydrophobic surface with negligible hysteresis. Langmuir 2013, 29, 10785-10795.

[23] Xu, X.; Ma, L. Analysis of the effects of evaporative cooling on the evaporation of liquid droplets using a combined field approach. Sci. Rep. 2015, 5, 8614.

[24] Nguyen, T. A. H.; Biggs, S. R.; Nguyen, A. V. Analytical model for diffusive evaporation of sessile droplets coupled with interfacial cooling effect. Langmuir 2018, 34, 6955-6962.

[25] Gleason, K.; Voota, H.; Putnam, S. A. Steady-state droplet evaporation: Contact angle influence on the evaporation efficiency. Int. J. Heat Mass Transfer. 2016, $101,418-426$.

[26] Dunn, G. J.; Wilson, S. K.; Duffy, B. R.; David, S.; Sefiane, K. The strong influence of substrate conductivity on droplet evaporation. J. Fluid Mech. 2009, $623,329-351$

[27] Wang, Y. L.; Ma, L. R.; Xu, X. F.; Luo, J.B. Combined effects of underlying substrate and evaporative cooling on the evaporation of sessile liquid droplets. Soft Matter. 2015, 11, 5632-5640.

[28] Saenz, P. J.; Sefiane K.; Kim J.; Matar O.K.; Valluri P. Evaporation of sessile drops: a three-dimensional approach. J. Fluid Mech. 2015, 772, 705-739.

[29] Petsi, A. J.; Burganos, V. N. Temperature distribution inside an evaporating two-dimensional droplet lying on curved or flat substrates. Phys. Rev. E. 2011, 84,011201 
[30] Dunn, G. J.; Wilson, S. K.; Duffy, B. R.; David, S; Sefiane, K. A mathematical model for the evaporation of a thin sessile liquid droplet: comparison between experiment and theory. Colloids Surf. A. 2008, 323, 50-55. 


\section{Figure captions}

Figure 1. Schematic diagram of a sessile evaporating droplet on curved substrate in toroidal coordinate

Figure 2. Comparison of total evaporation rates over different base radii between current theoretical model and experimental data at flat substrate $\left(\theta_{\text {sub }}=0\right)$ (Symbols are the experimental data from Dunn et al. [26, 30], solid lines are theoretical results from our model.)

Figure 3. The normalized temperature field within an evaporating droplet and substrate under different ratios $k_{R}$ of thermal conductivities of curved substrate over droplet at $E_{0}=1, \theta_{\mathrm{Sub}}=40^{\circ}$ and $\theta_{\mathrm{CA}}=50^{\circ}$

Figure 4. Radial variation of normalized evaporative fluxes, interfacial temperatures along the droplet surface under different ratios $k_{\mathrm{R}}$ of thermal conductivities of curved substrate over droplet at $E_{0}=1, \theta_{\mathrm{Sub}}=40^{\circ}$ and $\theta_{\mathrm{CA}}=50^{\circ}$

Figure 5. Radial variation of normalized heat fluxes, interfacial temperatures along the substrate surface under different ratios $k_{\mathrm{R}}$ of thermal conductivities of curved substrate over droplet at $E_{0}=1, \theta_{\mathrm{Sub}}=40^{\circ}$ and $\theta_{\mathrm{CA}}=50^{\circ}$

Figure 6. Variation of normalized evaporative fluxes $J$, interfacial temperatures $T$ along the droplet surface under different contact angles $\theta_{\mathrm{CA}}$ of droplet on curved substrate at $E_{0}=1, k_{\mathrm{R}}=0.1, \theta_{\text {Sub }}=40^{\circ}$

Figure 7.Transient variations of contact angle $\theta_{\mathrm{CA}}$ of droplet under different ratios $k_{\mathrm{R}}$ 
of thermal conductivities of curved substrate over droplet at $E_{0}=1, \theta_{\mathrm{Sub}}=40^{\circ}$ Figure 8.Transient variations of contact angle of droplet $\theta_{\mathrm{CA}}$ under different evaporative cooing number $E_{0}$ on curved substrate at $\theta_{\text {Sub }}=40^{\circ}$

Figure 9. Transient variations of contact angle of droplet $\theta_{\mathrm{CA}}$ under different initial contact angles $\theta_{\mathrm{In}}$ of the droplet on curved substrate at $E_{0}=1, \theta_{\mathrm{Sub}}=40^{\circ}$ Figure 10.Transient variations of contact angle of droplet $\theta_{\mathrm{CA}}$ under different tangential angles of curved substrate $\theta_{\text {Sub }}$ at $E_{0}=1$ 


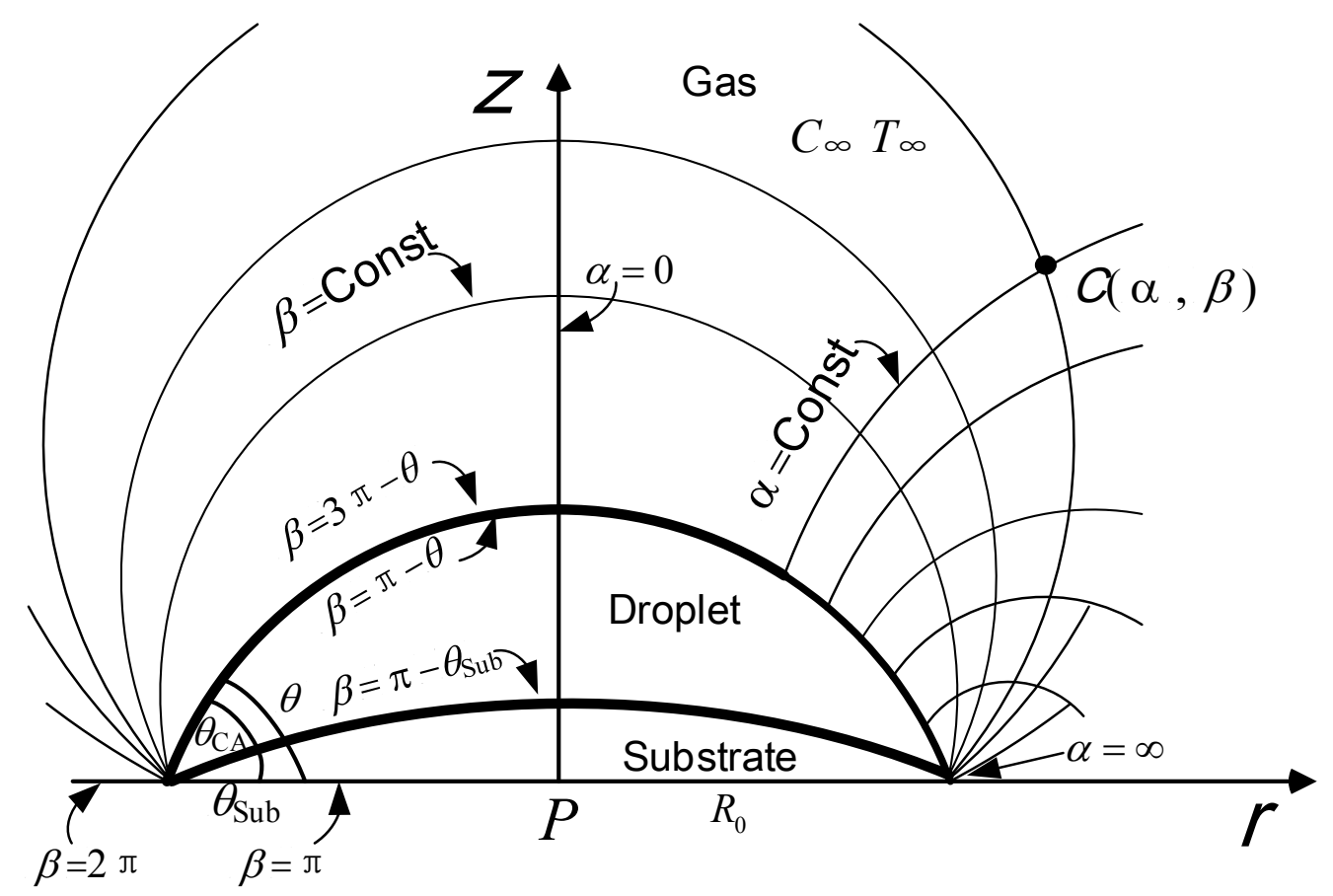

Figure 1. Schematic diagram of a sessile evaporating droplet on curved substrate in toroidal coordinate 


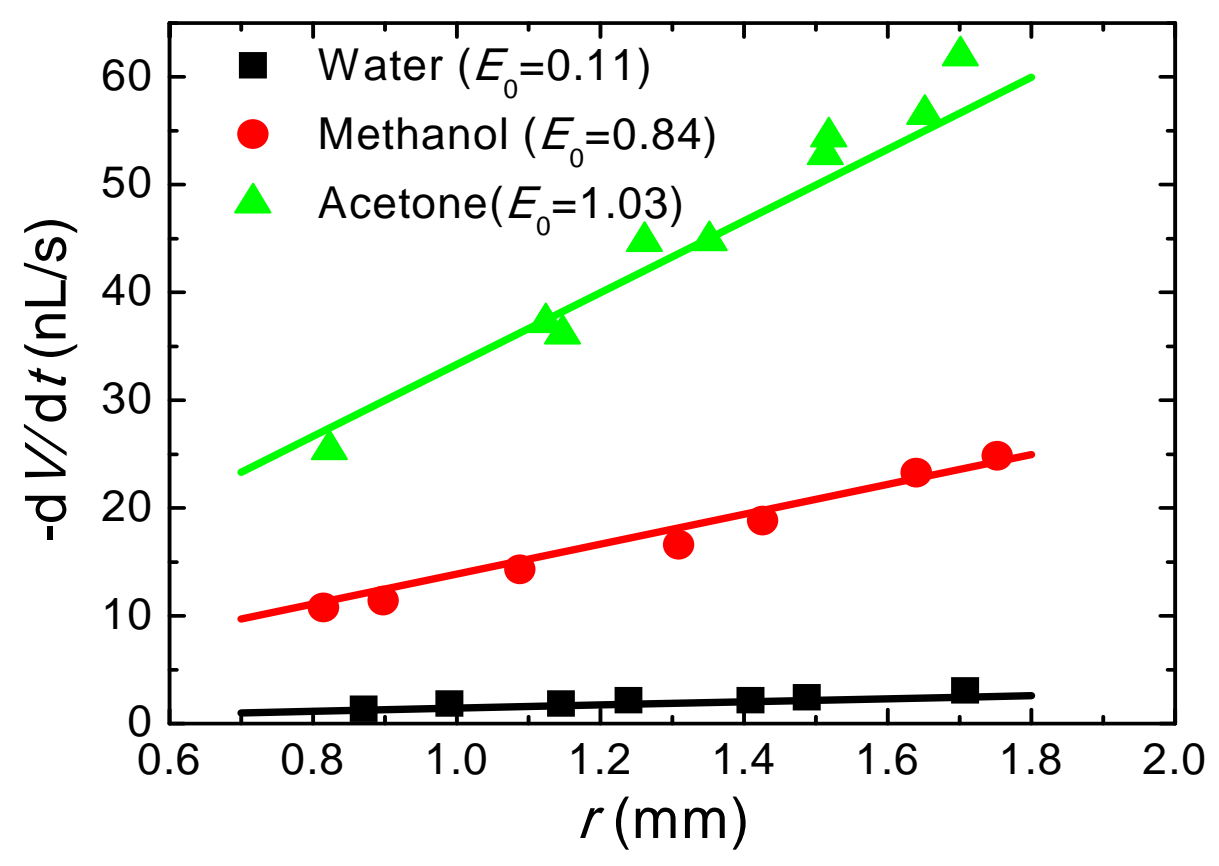

Figure 2. Comparison of total evaporation rates over different base radii between current theoretical model and experimental data at flat substrate $\left(\theta_{\text {sub }}=0\right)($ Symbols are the experimental data from Dunn et al. [26, 30], solid lines are theoretical results from our model.) 


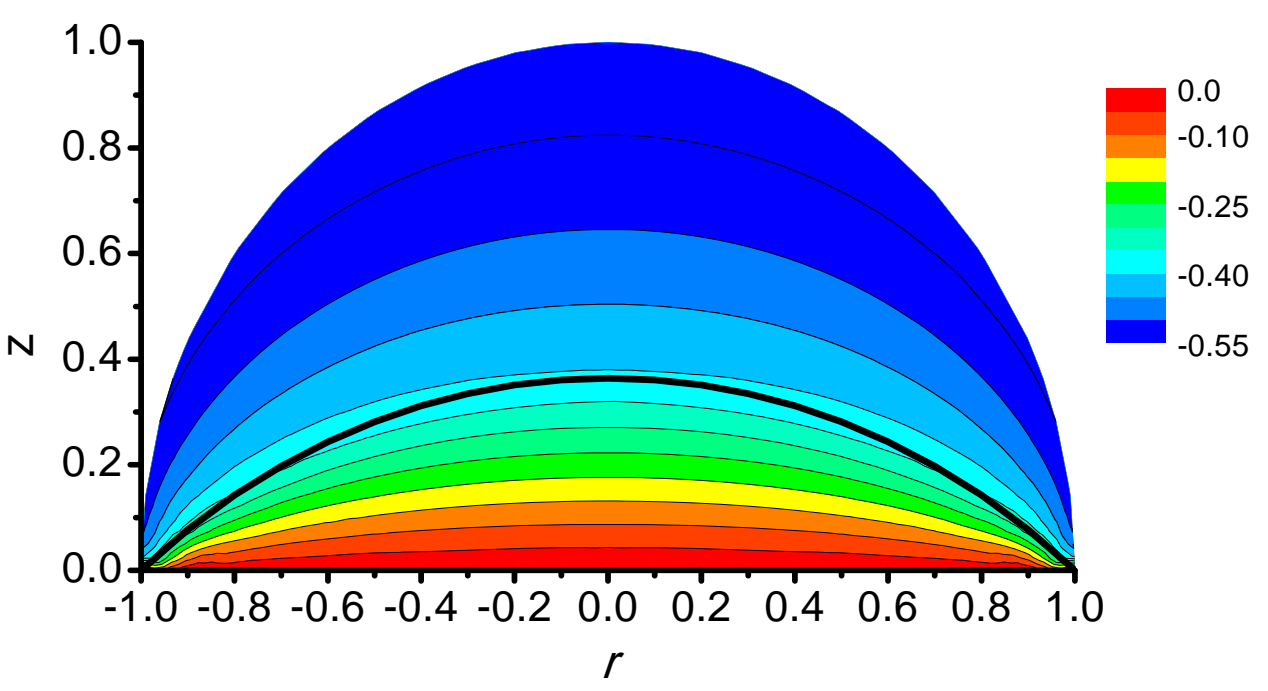

(a) $k_{R}=0.5$

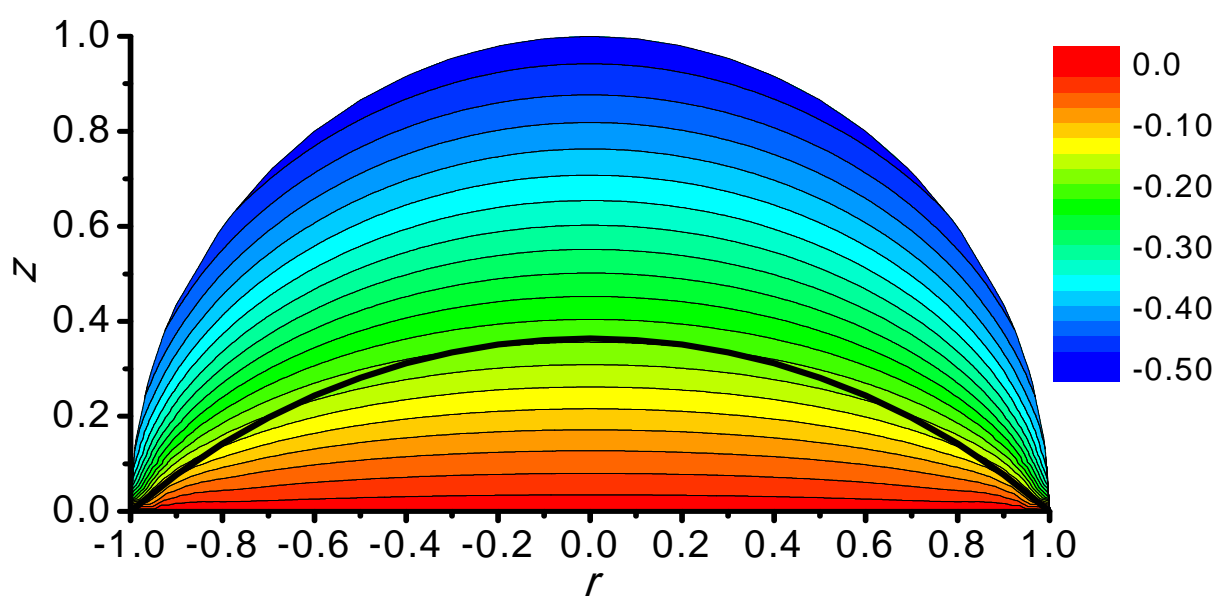

(b) $k_{R}=1$ 


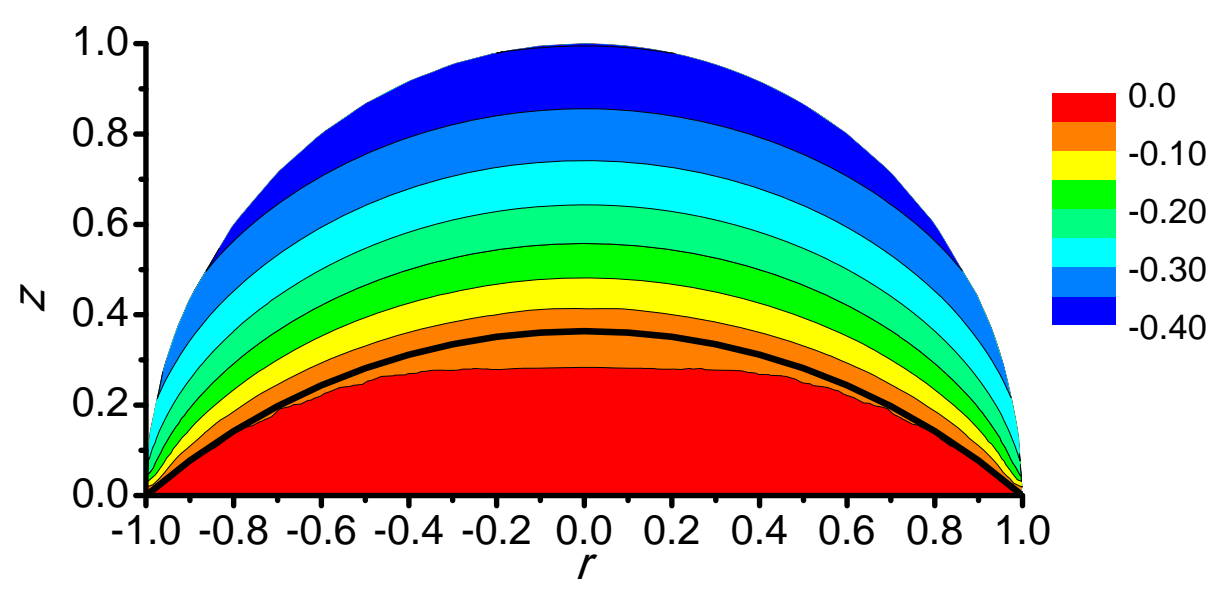

(c) $k_{R}=5$

Figure 3. The normalized temperature field within an evaporating droplet and substrate under different ratios $k_{R}$ of thermal conductivities of curved substrate over droplet at $E_{0}=1, \theta_{\mathrm{Sub}}=40^{\circ}$ and $\theta_{\mathrm{CA}}=50^{\circ}$ 


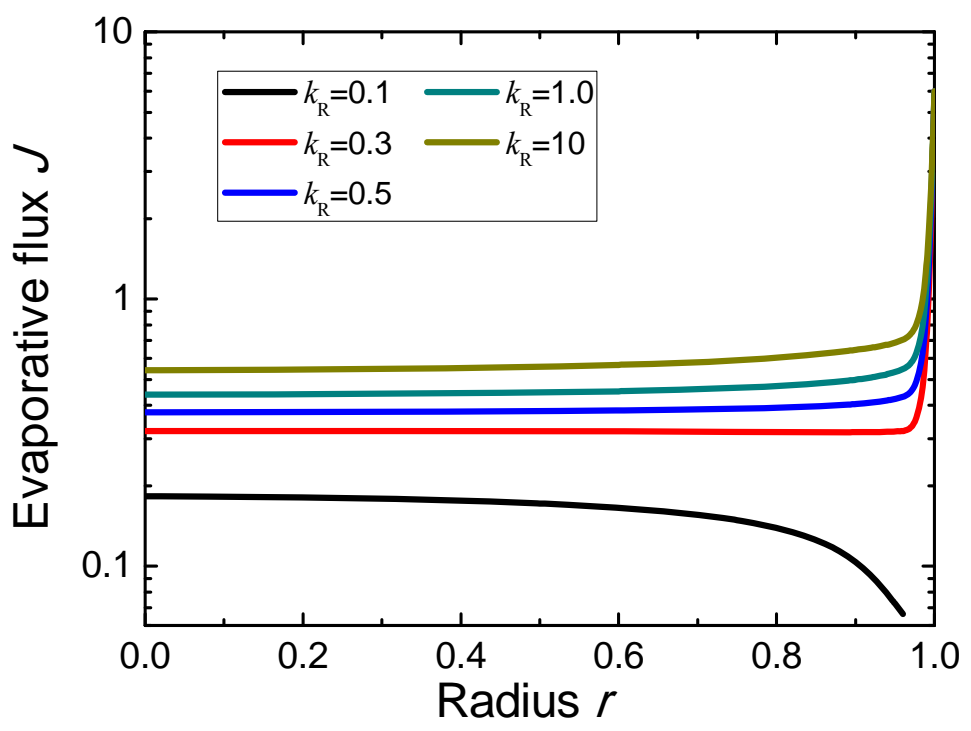

(a) Evaporative flux

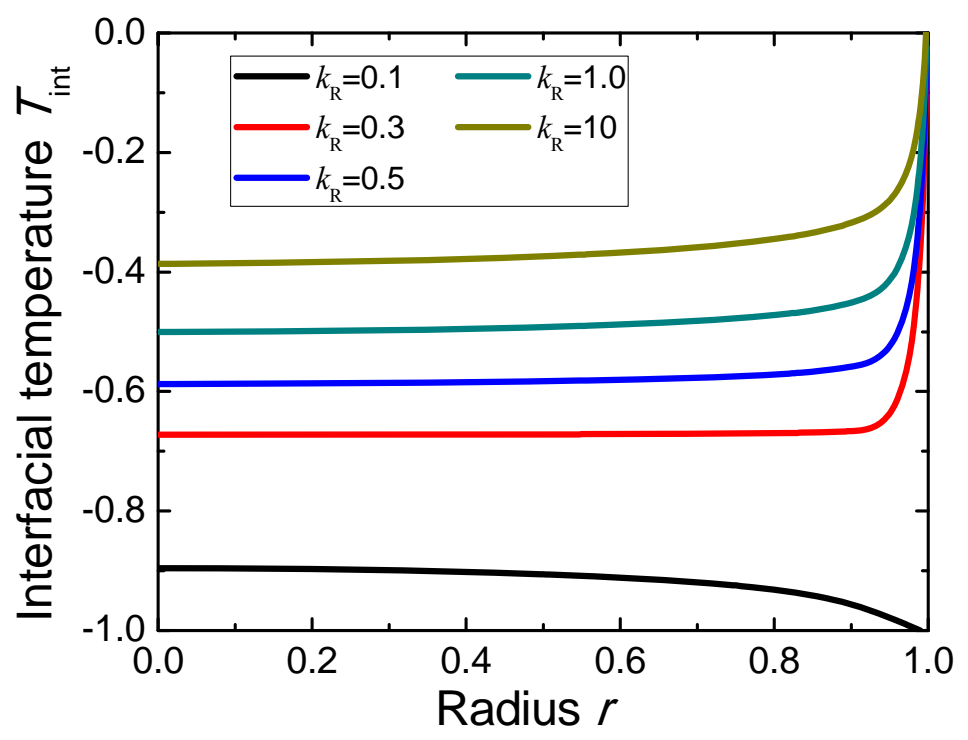

(b) Interfacial temperature

Figure 4. Radial variation of normalized evaporative fluxes, interfacial temperatures along the droplet surface under different ratios $k_{\mathrm{R}}$ of thermal conductivities of curved substrate over droplet at $E_{0}=1, \theta_{\text {Sub }}=40^{\circ}$ and $\theta_{\mathrm{CA}}=50^{\circ}$ 


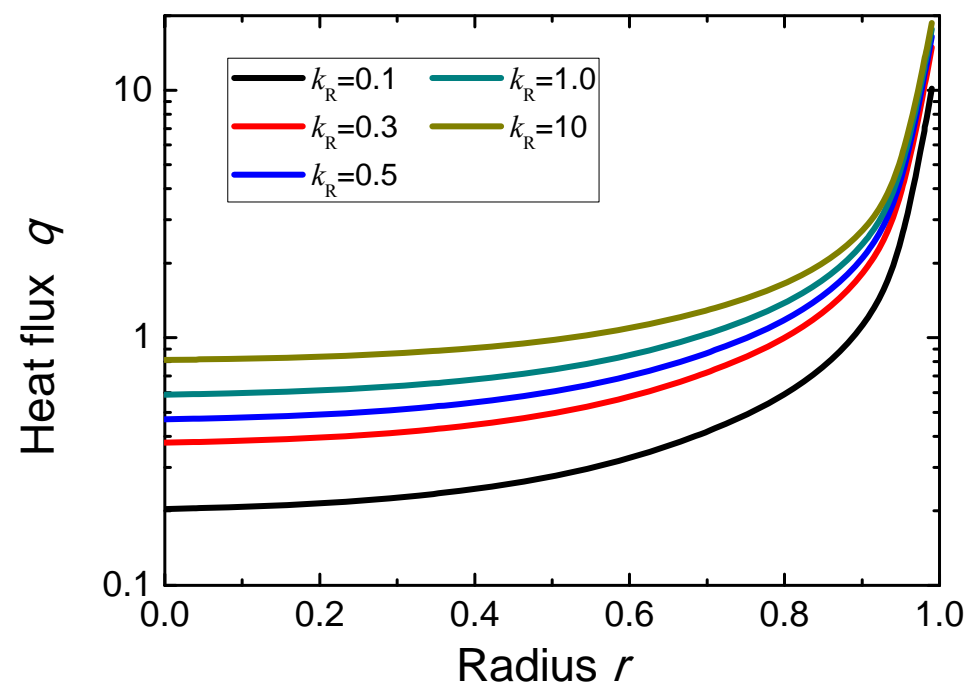

(a) Heat flux

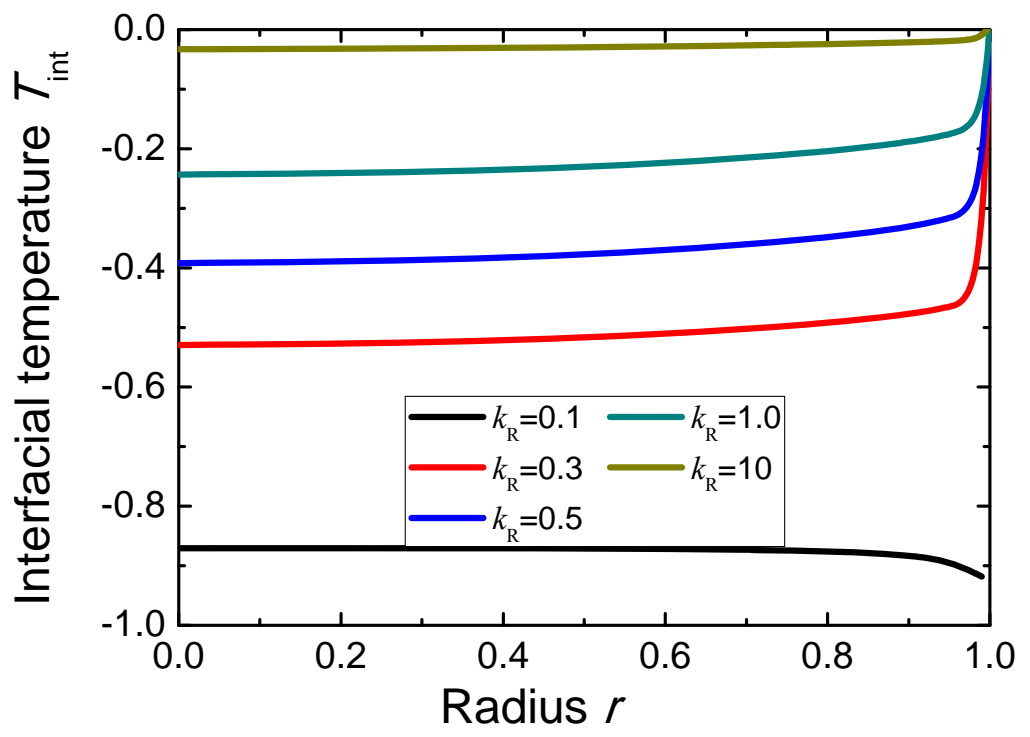

(b) Interfacial temperature

Figure 5. Radial variation of normalized heat fluxes, interfacial temperatures along the substrate surface under different ratios $k_{\mathrm{R}}$ of thermal conductivities of curved substrate over droplet at $E_{0}=1, \theta_{\text {Sub }}=40^{\circ}$ and $\theta_{\mathrm{CA}}=50^{\circ}$ 


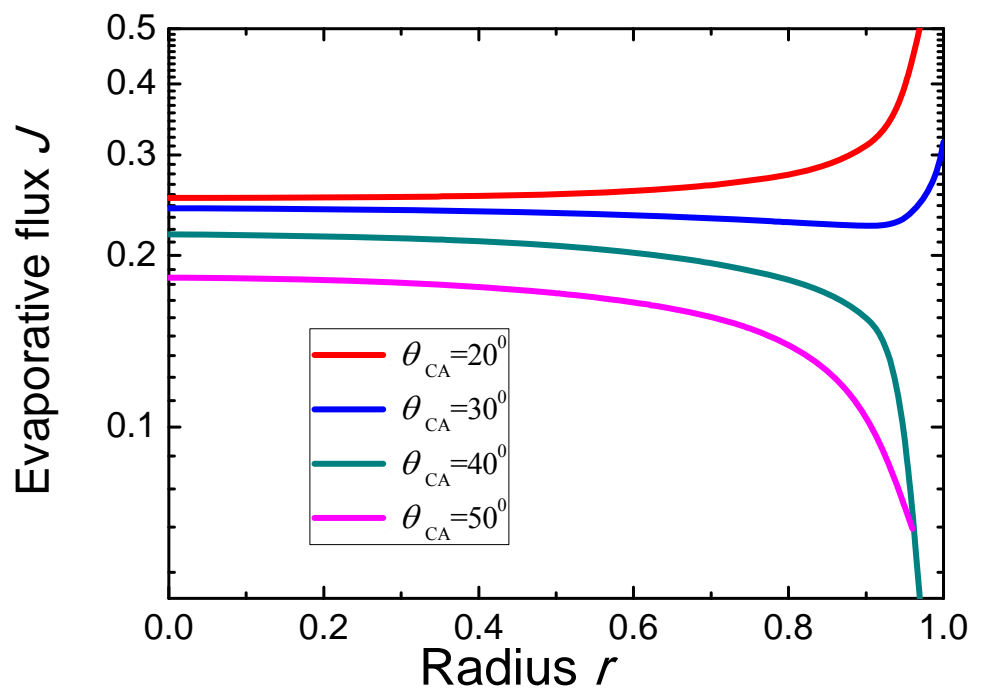

(a) Evaporative flux

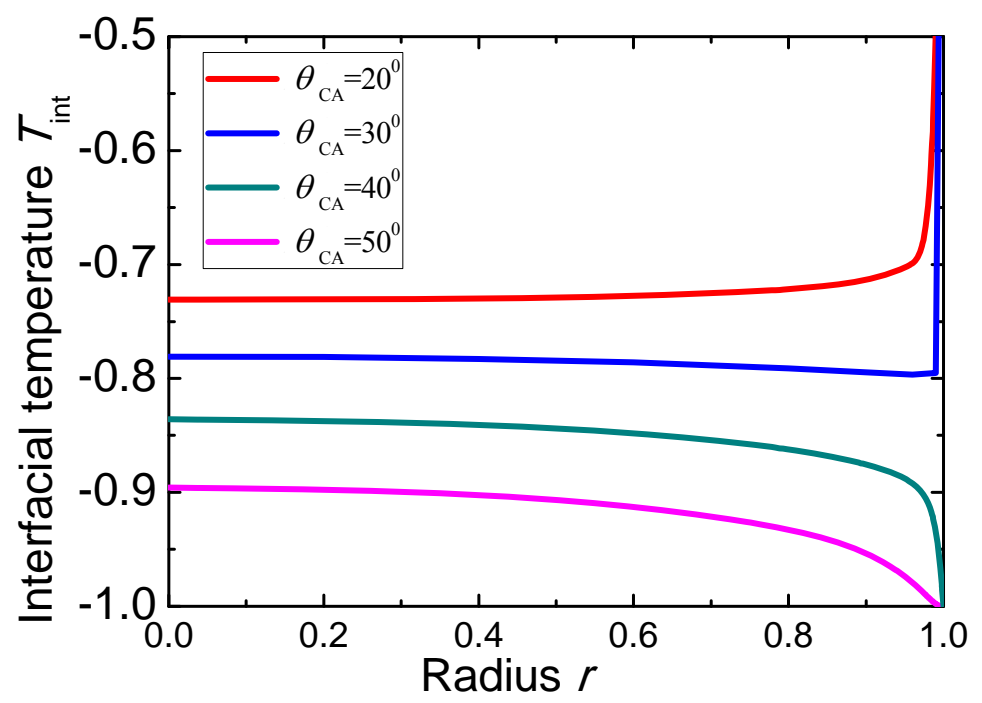

(b) Interfacial temperature

Figure 6. Variation of normalized evaporative fluxes $J$, interfacial temperatures $T$ along the droplet surface under different contact angles $\theta_{\mathrm{CA}}$ of droplet on curved substrate at $E_{0}=1, k_{\mathrm{R}}=0.1, \theta_{\text {Sub }}=40^{\circ}$ 


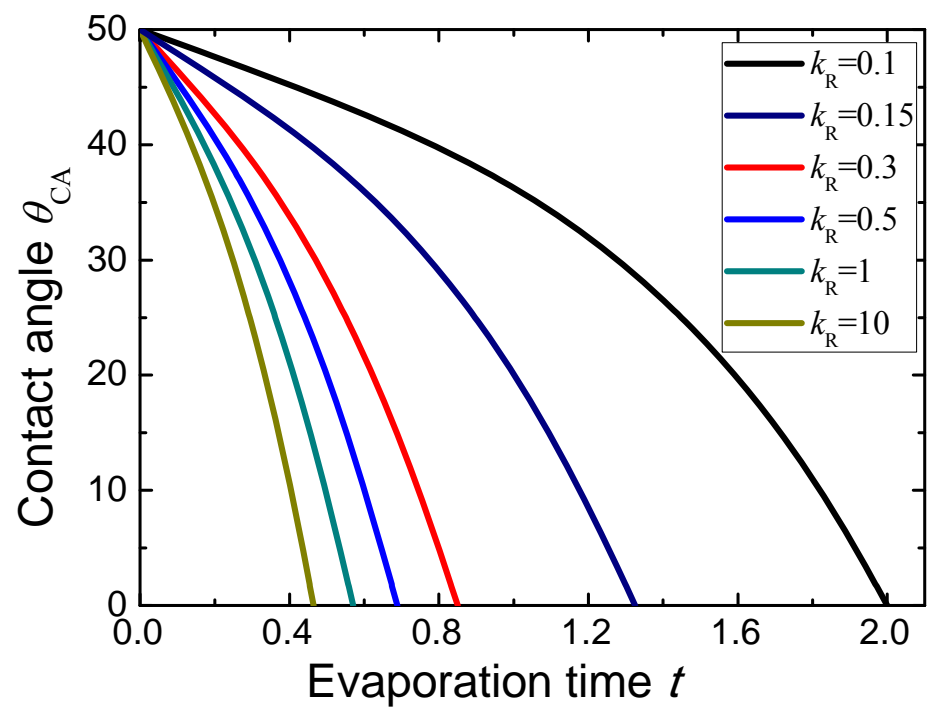

Figure 7.Transient variations of contact angle $\theta_{\mathrm{CA}}$ of droplet under different ratios $k_{\mathrm{R}}$ of thermal conductivities of curved substrate over droplet at $E_{0}=1, \theta_{\mathrm{Sub}}=40^{\circ}$ 


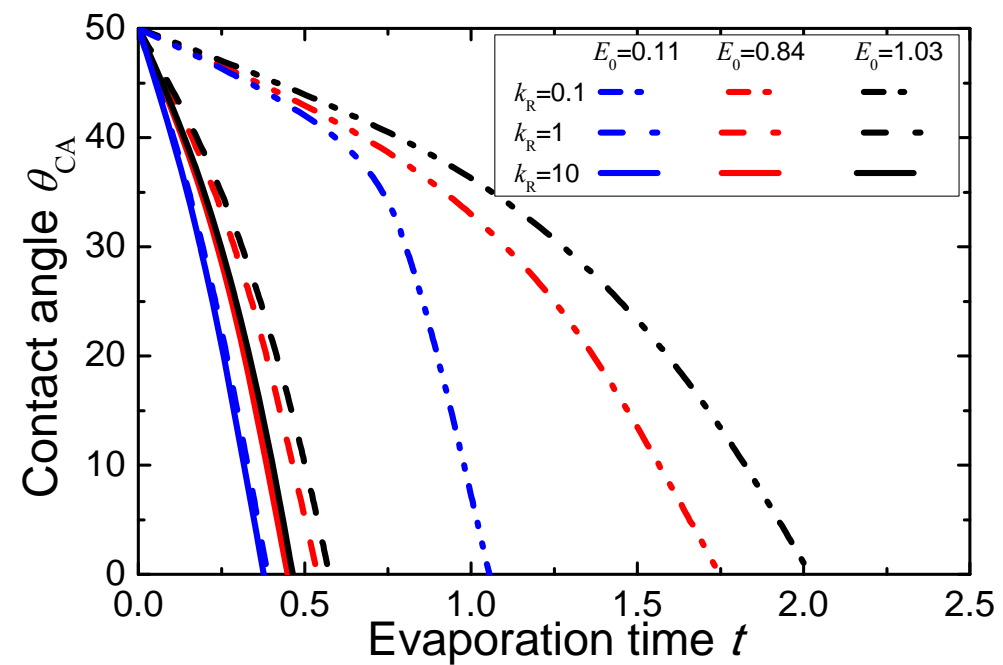

Figure 8. Transient variations of contact angle of droplet $\theta_{\mathrm{CA}}$ under different evaporative cooing number $E_{0}$ on curved substrate at $\theta_{\text {Sub }}=40^{\circ}$ 


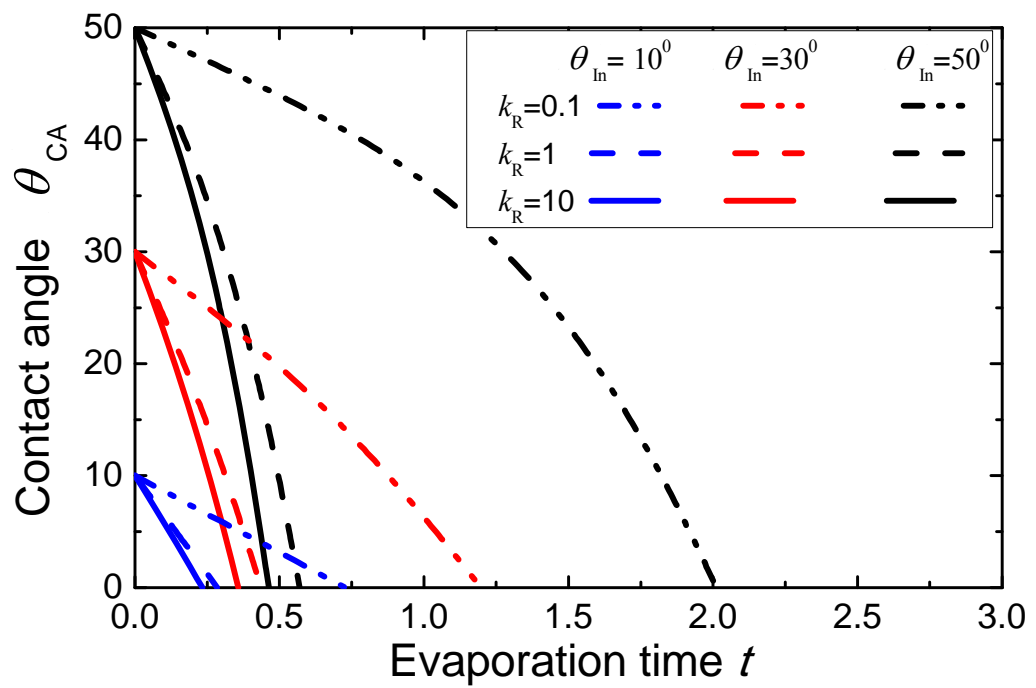

Figure 9. Transient variations of contact angle of droplet $\theta_{\mathrm{CA}}$ under different initial contact angles $\theta_{\mathrm{In}}$ of the droplet on curved substrate at $E_{0}=1, \theta_{\mathrm{Sub}}=40^{\circ}$ 


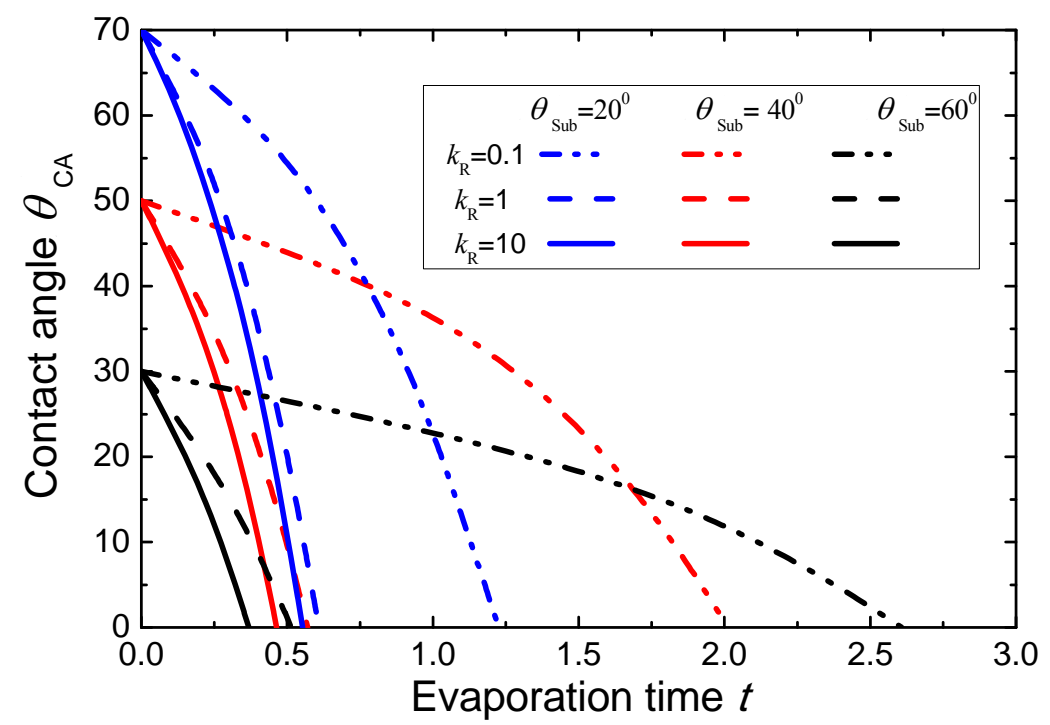

Figure 10.Transient variations of contact angle of droplet $\theta_{\mathrm{CA}}$ under different tangential angles of curved substrate $\theta_{\text {Sub }}$ at $E_{0}=1$ 